\title{
The Geometry of a $q$-Deformed Phase Space
}

\author{
B.L. Cerchiai ${ }^{1,2}$ R. Hinterding ${ }^{1,2}$ \\ J. Madore ${ }^{2,3}$, and J. Wess ${ }^{1,2}$ \\ ${ }^{1}$ Sektion Physik, Ludwig-Maximilian Universität, \\ Theresienstraße 37, D-80333 München \\ ${ }^{2}$ Max-Planck-Institut für Physik \\ (Werner-Heisenberg-Institut) \\ Föhringer Ring 6, D-80805 München \\ ${ }^{3}$ Laboratoire de Physique Théorique et Hautes Energies \\ Université de Paris-Sud, Bâtiment 211, F-91405 Orsay
}

\begin{abstract}
The geometry of the $q$-deformed line is studied. A real differential calculus is introduced and the associated algebra of forms represented on a Hilbert space. It is found that there is a natural metric with an associated linear connection which is of zero curvature. The metric, which is formally defined in terms of differential forms, is in this simple case identifiable as an observable.
\end{abstract}

LMU Preprint 98/08 


\section{Introduction and motivation}

There is a particularly simple noncommutative geometry, the 1-dimensional $q$-deformed euclidean space [10, 4, 11] $\mathbb{R}_{q}^{1}$ which can be completely analyzed from almost every point of view. Although this 'space' has but one 'dimension' and therefore there are no curvature effects, the corresponding algebra is most conveniently generated using elements which in the commutative limit correspond to coordinates in which the metric does not take its canonical flat form. If one writes, for example, the line element which describes the distance along the $y$-axis $d s^{2}=d y^{2}$ using the coordinate $x=e^{y}$ then one must write $d s^{2}=x^{-2} d x^{2}$; the metric has component $g_{11}=x^{-2}$. It has been argued previously [6] that to within a scale factor there is essentially a unique metric consistent with the noncommutative structure of an algebra. We shall see this clearly is the present example. We shall give a description of this metric in all detail since it is one of the rare cases in which the general formalism can be understood in terms of simple physical observables. In this section we shall give a brief review of the description of the differential structure of a noncommutative 'space' from the point of view of differential forms and from the 'dual' point of view of twisted derivations. In Section 2, after a few introductory remarks concerning the algebras $\mathbb{C}_{q}^{n}$ and $\mathbb{R}_{q}^{n}$ for general $n$, we describe the algebra $\mathbb{R}_{q}^{1}$. In Section 3 we introduce two conjugate differential calculi over this algebra and in Section 4 we propose a construction of a real differential calculus. In Section 5 we discuss a 'dual' point of view using twisted derivations. In Section 6 we briefly mention integration. In Section 7 we discuss the geometry of $\mathbb{R}_{q}^{1}$ using the unique local metric. In Section 8 we introduce Yang-Mills fields and in Section 9 we discuss the Schrödinger equation and the Klein-Gordon equation. In Section 10 we define an associated phase space [11] and briefly discuss the harmonic oscillator. The final section is devoted to a discussion of the effects of choosing an alternative non-local metric. Implicitly this metric has been used before [19].

Noncommutative geometry is geometry which is described by an associative algebra $\mathcal{A}$ which is usually but not essentially noncommutative and in which the set of points, if it exists at all, is relegated to a secondary role. For a thorough exposition of the subject we refer to the book by Connes [5]; for a gentle introduction we refer to Madore [21] or to Landi [18]. We shall be exclusively interested here in algebras which are in some sense deformations of algebras of smooth functions over a manifold. A differential calculus over $\mathcal{A}$ is another associative algebra $\Omega^{*}(\mathcal{A})$, with a differential $d$, which plays the role of the de Rham differential calculus and must tend to this calculus in the commutative limit. The differential calculus is what gives structure to the set of 'points'. It determines the 'dimension' for example. It would determine the number of nearest neighbours in the case of a lattice. Over a given $\mathcal{A}$ one can construct many differential calculi and the one which one choses depends evidently on the limit manifold one has in mind. There are many ways one can construct differential calculi. Historically the first construction [5] was based on an operator which played in some sense the role of the Dirac operator in ordinary geometry. This is extremely well suited to study the global aspects of geometries which in some sense resemble compact spaces with positive-definite metrics. To study noncommutative analogs of noncompact manifolds with metrics of arbitrary signature it is perhaps more practical to use calculi which are based on sets of derivations. We shall use this method here. In all cases the entire calculus can be considered as implicit in the module structure of the set of 1-forms. We shall consider only the cases where this module is free as a left or right module. It will in general not be free as a bimodule.

There are basically two points of view. One can start with a set of derivations in the strict sense of the word, a set of linear maps of the algebra into itself which satisfy 
the Leibniz rule and use them as basis for the construction of the associated differential forms. Or one can start with a set of differential forms obtained for example from some covariance criterion and construct a set of possibly twisted derivations which are dual to the forms. By 'twisted' here we mean derivations which satisfy a modified form of the Leibniz rule. We shall describe both points of view and compare them.

Let $\mathcal{A}$ be an algebra and $\lambda_{a}, 1 \leq a \leq n$ a set of $n$ elements of $\mathcal{A}$ which is such that only the identity commutes with it. This rule implies that only multiples of the identity will have a vanishing differential. We have obviously therewith excluded commutative algebras from consideration. In the example we consider this condition will not be satisfied, which explains why we can have a noncommutative geometry with only one dimension. We shall comment on this latter. We introduce a set of derivations $e_{a}$ defined on an arbitrary element $f \in \mathcal{A}$ by $e_{a} f=\left[\lambda_{a}, f\right]$. We have here given the $\lambda_{a}$ the physical dimensions of mass; we set this mass scale equal to one. Suppose that the algebra is generated formally by $n$ elements $x^{i}$. If one defines the differential of $f \in \mathcal{A}$ by $d f\left(e_{a}\right)=e_{a} f$ exactly as one does in ordinary geometry, or by any other method, then one finds that in general

$$
d x^{i}\left(e_{a}\right) \neq \delta_{a}^{i}
$$

The 'natural' basis $e_{a}$ of the derivations are almost never dual to the 'natural' basis $d x^{i}$ of the 1 -forms. There are basically two ways to remedy the above default. One can try to construct a new basis $\theta^{a}$ which is dual to the basis of the derivations or one can introduce derivations $\partial_{i}$ which satisfy a modified form of the Leibniz rule and which are dual to the $d x^{i}$. One has then either, or both, of the following equations:

$$
\theta^{a}\left(e_{b}\right)=\delta_{b}^{a}, \quad d x^{i}\left(\partial_{j}\right)=\delta_{j}^{i}
$$

In general these two points of view are equivalent. By construction the $\theta^{a}$ commute with all elements of the algebra. These commutation relations define the structure of the 1-forms as a bimodule over the algebra.

We recall briefly the construction based on derivations [6]. One finds that for the 'frame' or 'Stehbein' $\theta^{a}$ to exist the $\lambda_{a}$ must satisfy a constraint equation

$$
2 \lambda_{c} \lambda_{d} P_{a b}^{c d}-\lambda_{c} F_{a b}^{c}-K_{a b}=0
$$

with all the coefficients lying in the center $\mathcal{A}$. The first set of coefficients must be non-vanishing if the module of 2 -forms is to be nontrivial; it is related to a quantity which satisfies a sort of Yang-Baxter equation. Equation (1.1) gives to the set of $\lambda_{a}$ the form of a twisted Lie algebra with a central extension. It is obviously a very severe restriction. If the algebra is a *-algebra then the $\lambda_{a}$ must be antihermitian if the derivations are to be real. The involution can be extended to the general forms as well as to the tensor product of 1 -forms by introducing a set $J^{a b}{ }_{c d}$ of central elements. If one introduces a covariant derivative and requires that it be real then the left and right Leibniz rules are connected through the $J^{a b}{ }_{c d}$. If the $J^{a b}{ }_{c d}$ satisfy the Yang-Baxter equation then the extension of the covariant derivative to the tensor product of two 1 -forms is real. More details of this can be found elsewhere 13.

The dual point of view 28] consists in choosing the differentials $d x^{i}$ as the starting point and constructing from them a set of twisted derivations which satisfy a modified Leibniz rule. Although at first sight this method seems to be less general than the first, being normally restricted to quantum spaces invariant under the coaction of some quantum group, in fact, as we saw above, the quantum-group structure is more or less implicit also in the first approach in the form of the Yang-Baxter equation. The dual point of view has also the advantage in the fact that the twisted derivations can 
be given a bimodule structure and an associated phase space is perhaps more naturally constructed.

A metric on an algebra $\mathcal{A}$ can be defined [9] in terms of the 1-forms of a differential calculus $\Omega^{*}(\mathcal{A})$ as a bilinear map

$$
g: \Omega^{1}(\mathcal{A}) \otimes_{\mathcal{A}} \Omega^{1}(\mathcal{A}) \rightarrow \mathcal{A}
$$

or [2] in terms of the twisted derivations $\mathcal{X}$ as a bilinear map

$$
g^{\prime}: \mathcal{X} \otimes_{\mathcal{A}} \mathcal{X} \rightarrow \mathcal{A}
$$

We have distinguished here the two maps but in the case which interests us here they are essentially one and the same. In terms of the basis these equations can be written respectively as

$$
g\left(\theta^{a} \otimes \theta^{b}\right)=g^{a b}, \quad g^{\prime}\left(\partial_{i} \otimes \partial_{j}\right)=g_{i j}^{\prime}
$$

Since the $\theta^{a}$ commute with the elements of the algebra one sees from the sequence of identities

$$
f g^{a b}=g\left(f \theta^{a} \otimes \theta^{b}\right)=g\left(\theta^{a} \otimes \theta^{b} f\right)=g^{a b} f
$$

for arbitrary $f \in \mathbb{R}_{q}^{1}$ that the $g^{a b}$ must lie in the center of $\mathbb{R}_{q}^{1}$; they must be real numbers. Since the $\partial_{i}$ do not commute with the elements of the algebra one sees from the sequence of (in)equalities

$$
f g_{i j}^{\prime}=g^{\prime}\left(f \partial_{i} \otimes \partial_{j}\right) \neq g^{\prime}\left(\partial_{i} \otimes \partial_{j} f\right)=g_{i j}^{\prime} f
$$

for arbitrary $f \in \mathbb{R}_{q}^{1}$ that the $g_{i j}^{\prime}$ cannot lie in the center of $\mathbb{R}_{q}^{1}$. The commutation relations between $f$ and $g_{i j}^{\prime}$ are however in principle calculable in terms of the commutation relations between $f$ and $\partial_{i}$. A more detailed exposition of the geometry of the algebra $\mathbb{R}_{q}^{3}$ has been given elsewhere 14.

Suppose that one particular 'coordinate' $x^{i}$ has a discrete spectrum $|k\rangle$. Then it is possible to give an observational definition of the distance $d s(k)$ between $|k\rangle$ and $|k+1\rangle$ in terms of $g$ or $g^{\prime}$ by identifying $d x$ as the difference between the two corresponding eigenvalues. It is our main purpose to study this identification in detail in a particularly simple case.

\section{The $q$-deformed euclidean spaces}

The $q$-deformed euclidean spaces [10] $\mathbb{C}_{q}^{n}$ and $\mathbb{R}_{q}^{n}$ are algebras which are covariant under the coaction of the quantum groups $S O_{q}(n)$. To describe them it is convenient to introduce the projector decomposition of the corresponding braid matrix

$$
\hat{R}=q P_{s}-q^{-1} P_{a}+q^{1-n} P_{t}
$$

where the $P_{s}, P_{a}, P_{t}$ are $S O_{q}(n, \mathbb{R})$-covariant $q$-deformations of respectively the symmetric trace-free, antisymmetric and trace projectors. They are mutually orthogonal and their sum is equal to the identity:

$$
P_{s}+P_{a}+P_{t}=1
$$

The trace projector is 1-dimensional and its matrix elements can be written in the form

$$
P_{t}^{i j} k l=\left(g^{m n} g_{m n}\right)^{-1} g^{i j} g_{k l},
$$


where $g_{i j}$ is the $q$-deformed euclidean metric. The $q$-euclidean space is the formal associative algebra $\mathbb{C}_{q}^{n}$ with generators $x^{i}$ and relations

$$
P_{a}^{i j}{ }_{k l} x^{k} x^{l}=0
$$

for all $i, j$. One obtains the real $q$-euclidean space by choosing $q \in \mathbb{R}^{+}$and by giving the algebra an involution defined by

$$
x_{i}^{*}=x^{j} g_{j i}
$$

This condition is an $S O_{q}(n, \mathbb{R})$-covariant condition and $n$ linearly independent, real coordinates can be obtained as combinations of the $x^{i}$. The 'length' squared

$$
r^{2}:=g_{i j} x^{i} x^{j}=x_{i}^{*} x^{i}
$$

is $S O_{q}(n, \mathbb{R})$-invariant, real and generates the center $\mathcal{Z}\left(\mathbb{R}_{q}^{n}\right)$ of $\mathbb{R}_{q}^{n}$. We can extend $\mathbb{R}_{q}^{n}$ by adding to it the square root $r$ of $r^{2}$ and the inverse $r^{-1}$. For reasons to become clear below when we introduce differential calculi over $\mathbb{R}_{q}^{n}$ we add also an extra generator $\Lambda$ called the dilatator and its inverse $\Lambda^{-1}$ chosen such that

$$
x^{i} \Lambda=q \Lambda x^{i} .
$$

We shall choose $\Lambda$ to be unitary. Since $r$ and $\Lambda$ do not commute the center of the new extension is trivial.

We shall be here interested only in the case $n=1$. The algebra $\mathbb{R}_{q}^{1}$ has only two generators $x$ and $\Lambda$ which satisfy the commutation relation $x \Lambda=q \Lambda x$. We shall choose $x$ hermitian and $q \in \mathbb{R}^{+}$with $q>1$. This is a modified version of the Weyl algebra with $q$ real instead of with unit modulus. We can represent the algebra on a Hilbert space $\mathcal{R}_{q}$ with basis $|k\rangle$ by

$$
x|k\rangle=q^{k}|k\rangle, \quad \Lambda|k\rangle=|k+1\rangle .
$$

This is an infinite-dimensional version of the basis introduced by Schwinger [26] to study the Weyl algebra when $q$ is a root of unity. It explains the origin of the expression 'dilatator'. Contrary to the case considered by Schwinger however the spectrum of $\Lambda$ is continuous.

Introduce the element $y$ by the action

$$
y|k\rangle=k|k\rangle
$$

on the basis elements. Then the commutation relations between $\Lambda$ and $y$ can be written as

$$
\Lambda^{-1} y \Lambda=y+1 \text {. }
$$

We can write $x=q^{y}$ as an equality within $\mathbb{R}_{q}^{1}$. We shall on occasion renormalize y. We introduce a renormalization parameter $z$ as

$$
z=q^{-1}(q-1)>0 .
$$

The renormalization is then given by the substitution

$$
z y \mapsto y
$$

With the new value of $y$ the spacing between the spectral lines vanishes with $z$. We shall refer to the old units as Planck units and the new ones as laboratory units. One can show [22] that the von Neumann algebra generated by $\Lambda$ and $x$ or $y$ is a factor of type $I_{\infty}$. 


\section{The $q$-deformed calculi}

One possible differential calculus over the algebra $\mathbb{R}_{q}^{1}$ is constructed by setting $d \Lambda=0$ and

$$
x d x=d x x, \quad d x \Lambda=q \Lambda d x .
$$

The frame is given by $\theta^{1}=x^{-1} d x$. This calculus has an involution given by $(d x)^{*}=d x^{*}$ but it is not based on derivations and it has no covariance properties with respect to $S O_{q}(1)$.

We consider therefore another differential calculus $\Omega^{*}\left(\mathbb{R}_{q}^{1}\right)$ based on the relations 3

$$
x d x=q d x x, \quad d x \Lambda=q \Lambda d x
$$

for the 1-forms. If we choose

$$
\lambda_{1}=-z^{-1} \Lambda
$$

then

$$
e_{1} x=q \Lambda x, \quad e_{1} \Lambda=0
$$

and the calculus (3.1) is defined by the condition $d f\left(e_{1}\right)=e_{1} f$ for arbitrary $f \in \mathbb{R}_{q}^{1}$. By setting

$$
\lambda_{2}=z^{-1} x
$$

and introducing a second derivation

$$
e_{2} \Lambda=q \Lambda x, \quad e_{2} x=0
$$

one could extend the calculus (3.1) by the condition $d f\left(e_{2}\right)=e_{2} f$ for arbitrary $f \in \mathbb{R}_{q}^{1}$. One would find $x d \Lambda=q d \Lambda x$. We shall not do so since it will be seen that $\Lambda$ is in a sense an element of the phase space associated to $x$ and we are interested in position-space geometry.

The adjoint derivation $e_{1}^{\dagger}$ of $e_{1}$ is defined by

$$
e_{1}^{\dagger} f=\left(e_{1} f^{*}\right)^{*}
$$

The $e_{1}^{\dagger}$ on the left-hand side is not an adjoint of an operator $e_{1}$. It is defined uniquely in terms of the involution of $\mathbb{R}_{q}^{1}$ whereas $e_{1}$ acts on this algebra as a vector space.

Since $\Lambda$ is unitary we have $\left(\lambda_{1}\right)^{*} \neq-\lambda_{1}$ and $e_{1}$ is not a real derivation. We introduce [3] therefore a second differential calculus $\bar{\Omega}^{*}\left(\mathbb{R}_{q}^{1}\right)$ defined by the relations

$$
x \bar{d} x=q^{-1} \bar{d} x x, \quad \bar{d} x \Lambda=q \Lambda \bar{d} x
$$

and based on the derivation $\bar{e}_{1}$ formed using $\bar{\lambda}_{1}=-\lambda_{1}^{*}$. This calculus is defined by the condition $\bar{d} f\left(\bar{e}_{1}\right)=\bar{e}_{1} f$ for arbitrary $f \in \mathbb{R}_{q}^{1}$. The derivation $\bar{e}_{1}$ is also not real. It is easy to see however that

$$
e_{1}^{\dagger}=\bar{e}_{1}
$$

and therefore that $(d f)^{*}=\bar{d} f^{*}$. By simple induction we find that for arbitrary integer $n$

$$
e_{1} x^{n}=z^{-1}\left(q^{n}-1\right) \Lambda x^{n}, \quad \bar{e}_{1} x^{n}=z^{-1}\left(1-q^{-n}\right) \Lambda^{-1} x^{n} .
$$

We can represent also $\Omega^{*}\left(\mathbb{R}_{q}^{1}\right)$ and $\bar{\Omega}^{*}\left(\mathbb{R}_{q}^{1}\right)$ on $\mathcal{R}_{q}$. For the two elements $d x$ and $\bar{d} x$ we have respectively

$$
d x|k\rangle=\alpha q^{k+1}|k+1\rangle, \quad \bar{d} x|k\rangle=\bar{\alpha} q^{k}|k-1\rangle
$$


with two arbitrary complex parameters $\alpha$ and $\bar{\alpha}$. One sees that $(d x)^{*}=\bar{d} x$ if and only if $\alpha^{*}=\bar{\alpha}$. It is possible to represent $d$ and $\bar{d}$ as the operators

$$
d=-z^{-1} \alpha \operatorname{ad} \Lambda, \quad \bar{d}=z^{-1} \bar{\alpha} \operatorname{ad} \Lambda^{-1} .
$$

It is easy to see that the commutation relations (3.1) and (3.2) are satisfied. The above representations are certainly not unique [23].

The frame elements $\theta^{1}$ and $\bar{\theta}^{1}$ dual to the derivations $e_{1}$ and $\bar{e}_{1}$ are given by

$$
\begin{array}{ll}
\theta^{1}=\theta_{1}^{1} d x, & \theta_{1}^{1}=\Lambda^{-1} x^{-1}, \\
\bar{\theta}^{1}=\bar{\theta}_{1}^{1} \bar{d} x, & \bar{\theta}_{1}^{1}=q^{-1} \Lambda x^{-1} .
\end{array}
$$

On $\mathcal{R}_{q}$ they become the operators

$$
\theta^{1}=\alpha, \quad \bar{\theta}^{1}=\bar{\alpha}
$$

proportional to the unit element. They were so constructed. The algebra $\mathbb{R}_{q}^{1}$ is a subalgebra of the graded algebra of forms $\Omega^{*}\left(\mathbb{R}_{q}^{1}\right)$ and the representation (2.2) can be extended to a representation of the latter. In fact since $\Omega^{1}\left(\mathbb{R}_{q}^{1}\right)$ and $\bar{\Omega}^{1}\left(\mathbb{R}_{q}^{1}\right)$ are free $\mathbb{R}_{q^{1}}^{1}$-modules of rank one with respectively the special basis $\theta^{1}$ and $\bar{\theta}^{1}$ we can identify

$$
\Omega^{*}\left(\mathbb{R}_{q}^{1}\right)=\bigwedge^{*} \otimes \mathbb{R}_{q}^{1}, \quad \bar{\Omega}^{*}\left(\mathbb{R}_{q}^{1}\right)=\bigwedge^{*} \otimes \mathbb{R}_{q}^{1}
$$

where $\bigwedge^{*}$ is the exterior algebra over $\mathbb{C}^{1}$ and so the extension is trivial.

From the two differential calculi $\Omega^{*}\left(\mathbb{R}_{q}^{1}\right)$ and $\bar{\Omega}^{*}\left(\mathbb{R}_{q}^{1}\right)$ we would like to construct a real differential calculus $\Omega_{R}^{*}\left(\mathbb{R}_{q}^{1}\right)$ with a differential $d_{R}$ such that $\left(d_{R} f\right)^{*}=d_{R} f^{*}$. The construction has nothing to do with the structure of $\mathbb{R}_{q}^{1}$ so we give it in terms of a general algebra $\mathcal{A}$.

\section{A Real calculus}

Consider an algebra $\mathcal{A}$ with involution over which there are two differential calculi $\left(\Omega^{*}(\mathcal{A}), d\right)$ and $\left(\bar{\Omega}^{*}(\mathcal{A}), \bar{d}\right)$ neither of which is necessarily real. Consider the product algebra $\tilde{\mathcal{A}}=\mathcal{A} \times \mathcal{A}$ and over $\tilde{\mathcal{A}}$ the differential calculus

$$
\tilde{\Omega}^{*}(\tilde{\mathcal{A}})=\Omega^{*}(\mathcal{A}) \times \bar{\Omega}^{*}(\mathcal{A}) .
$$

It has a natural differential given by $\tilde{d}=(d, \bar{d})$. The embedding

$$
\mathcal{A} \hookrightarrow \tilde{\mathcal{A}}
$$

given by $f \mapsto(f, f)$ is well defined and compatible with the involution

$$
(f, g)^{*}=\left(g^{*}, f^{*}\right)
$$

on $\tilde{\mathcal{A}}$.

Let $X$ and $\bar{X}$ be two derivations of $\mathcal{A}$. Then $\tilde{X}=(X, \bar{X})$ is a derivation of $\tilde{\mathcal{A}}$. We recall that a derivation $X$ of an algebra $\mathcal{A}$ is real if for arbitrary $f \in \mathcal{A}$ we have $X f^{*}=(X f)^{*}$. We saw in the previous section that $e_{1}$ and $\bar{e}_{1}$ are not real. Then $\tilde{X}$ is a real derivation if

$$
\tilde{X}(f, g))^{*}=(\tilde{X}(f, g))^{*} .
$$


This can be written as the conditions

$$
\bar{X} f^{*}=(X f)^{*}, \quad X g^{*}=(\bar{X} g)^{*} .
$$

The essential point to notice is that $\mathcal{A}$ does not necessarily remain invariant under real derivations of $\tilde{\mathcal{A}}$. This is to be expected since if $\mathcal{A}$ had 'interesting' real derivations they could be used to construct directly a real differential calculus over $\mathcal{A}$.

Suppose that $\Omega^{*}(\mathcal{A})$ is defined in term of a set of inner derivations $e_{a}=\operatorname{ad} \lambda_{a}$ and that $\bar{\Omega}^{*}(\mathcal{A})$ is defined in term of a set of inner derivations $\bar{e}_{a}=\operatorname{ad} \bar{\lambda}$. Suppose also that the corresponding $\tilde{e}_{a}=\left(e_{a}, \bar{e}_{a}\right)$ are real derivations of $\tilde{\mathcal{A}}$. From (4.3) we see that this will be the case if and only if $\bar{\lambda}_{a}=-\lambda_{a}^{*}$. We saw in the previous section that $\tilde{e}_{1}=\left(e_{1}, \bar{e}_{1}\right)$ is real and that in fact $\bar{\lambda}_{1}=-\lambda_{1}^{*}$. We define an involution on $\tilde{\Omega}^{*}(\tilde{\mathcal{A}})$ by the condition

$$
(\tilde{d}(f, g))^{*}\left(\tilde{e}_{a}\right)=\left(\tilde{e}_{a}(f, g)\right)^{*}=\tilde{e}_{a}\left(g^{*}, f^{*}\right) .
$$

The differential $\tilde{d}$ is real by construction [13].

Define $\mathcal{A}_{R}$ to be the smallest algebra which contains $\mathcal{A}$ and which is stable under the action of the derivations $\tilde{e}_{a}$. The image in $\tilde{\mathcal{A}}$ of the commutative subalgebra $\mathcal{A}_{0} \subset \mathcal{A}$ of $\mathcal{A}$ generated by $x$ is invariant under the involution (4.2). Define $e_{R a}$ to be the restriction of $\tilde{e}_{a}$ to $\mathcal{A}_{R}$ and $d_{R}$ to be the restriction of $\tilde{d}$ to $\mathcal{A}_{R}$. We have then

$$
d_{R} f\left(e_{R a}\right)=\left(e_{a} f, \bar{e}_{a} f\right)
$$

and $d_{R}$ is also real. We define

$$
\Omega_{R}^{1}(\mathcal{A}) \subset \tilde{\Omega}^{1}(\tilde{\mathcal{A}})
$$

to be the $\mathcal{A}_{R}$-bimodule generated by the image of $d_{R}$. We write $\Omega_{R}^{1}(\mathcal{A})$ instead of $\Omega_{R}^{1}\left(\mathcal{A}_{R}\right)$ since we keep $\Omega_{R}^{0}(\mathcal{A})=\mathcal{A}$. The module structure determines a differential calculus $\left(\Omega_{R}^{*}(\mathcal{A}), d_{R}\right)$. Suppose there exists a frame $\theta^{a}$ for $\Omega^{*}(\mathcal{A})$ and a frame $\bar{\theta}^{a}$ for $\bar{\Omega}^{*}(\mathcal{A})$. We can extend also the involution $(4.2)$ to all of $\Omega_{R}^{*}(\mathcal{A})$ by setting

$$
\left(\theta^{a}\right)^{*}=\bar{\theta}^{a}
$$

and we can define $\Omega_{R}^{1}(\mathcal{A})$ to be the $\mathcal{A}_{R}$ module generated by

$$
\theta_{R}^{a}=\left(\theta^{a}, \bar{\theta}^{a}\right)
$$

This is consistent with the previous definition since

$$
d_{R} f=e_{R a} f \theta_{R}^{a}, \quad e_{R a} f \in \mathcal{A}_{R}
$$

From the relations

$$
\begin{array}{ll}
\theta^{a}\left(e_{b}\right)=\delta_{b}^{a}, & \theta^{a}\left(\bar{e}_{b}\right)=0, \\
\bar{\theta}^{a}\left(e_{b}\right)=0, & \bar{\theta}^{1}\left(\bar{e}_{b}\right)=\delta_{b}^{a}
\end{array}
$$

it follows that the frame dual to the derivation $e_{R a}$ is indeed $\theta_{R}^{a}$ :

$$
\theta_{R}^{a}\left(e_{R b}\right)=\delta_{b}^{a}
$$

If we define the 'Dirac operators'

$$
\theta=-\lambda_{a} \theta^{a}, \quad \bar{\theta}=-\bar{\lambda}_{a} \bar{\theta}^{a}, \quad \theta_{R}=-\lambda_{R a} \theta_{R}^{a}
$$

then we find from the Equation (4.4) that for all $f \in \mathcal{A}$

$$
d f=-[\theta, f], \quad \bar{d} \bar{f}=-[\bar{\theta}, f], \quad d_{R} f=-\left[\theta_{R}, f\right] .
$$


Except for $\Omega_{R}^{0}(\mathcal{A})=\mathcal{A}$ we can write

$$
\Omega_{R}^{*}(\mathcal{A})=\bigwedge^{*} \otimes \mathcal{A}_{R}
$$

where $\Lambda^{*}$ is the algebra over $\mathbb{C}$ generated by the $\theta_{R}^{a}$.

We are now in a position to construct a real differential calculus over $\mathbb{R}_{q}^{1}$. According to the general remarks we see that $e_{R 1}=\left(e_{1}, \bar{e}_{1}\right)$ is a real derivation of $\mathbb{R}_{q R}^{1}$ and and that it is inner

$$
e_{R 1}=\operatorname{ad} \lambda_{R 1} \quad \lambda_{R 1}=\left(\lambda_{1}, \bar{\lambda}_{1}\right)=z^{-1}\left(-\Lambda, \Lambda^{-1}\right) .
$$

Because of the identity

$$
e_{R 1} x=\left(q \Lambda, \Lambda^{-1}\right) x
$$

we conclude that

$$
x d_{R} x=\left(q, q^{-1}\right) d_{R} x, \quad d_{R} x \Lambda=q \Lambda d_{R} x .
$$

These are the real-calculus equivalent of the relations (3.1) and (3.2). A representation of the 1 -forms of the differential calculus $\Omega_{R}^{*}\left(\mathbb{R}_{q}^{1}\right)$ can be given on the $\operatorname{direct} \operatorname{sum} \mathcal{R}_{q} \oplus \mathcal{R}_{q}$ of two separate and distinct copies of $\mathcal{R}_{q}$, one for $d x$ and one for $\bar{d} x$. From (3.4) one sees that $d_{R} x$ can be represented by the operator

$$
d_{R} x|k\rangle=q^{k}(q \alpha|k+1\rangle+\bar{\alpha} \overline{|k-1\rangle}) .
$$

We have placed a bar over the second term to underline the fact that it belongs to the second copy of $\mathcal{R}_{q}$.

Since the Equations (4.6) involve (in the case $\mathcal{A}=\mathbb{R}_{q}^{1}$ ) $e_{1}$ and $\bar{e}_{1}$ considered as derivations they cannot be implemented on $\mathcal{R}_{q}$. However $e_{1}$ and $\bar{e}_{1}$ can be considered as 'annihilation' operators which map $\Omega_{R}^{1}\left(\mathbb{R}_{q}^{1}\right)$ into $\Omega_{R}^{0}\left(\mathbb{R}_{q}^{1}\right)$. Similarly $\theta^{1}$ and $\bar{\theta}^{1}$ have an interpretation [16] as 'creation' operators which take $\Omega_{R}^{1}\left(\mathbb{R}_{q}^{1}\right)$ into $\Omega_{R}^{2}\left(\mathbb{R}_{q}^{1}\right) \equiv 0$. On $\mathcal{R}_{q} \oplus \mathcal{R}_{q}$ the involution is given by the map $\alpha \mapsto \bar{\alpha}$. We shall choose

$$
\alpha=1, \quad \bar{\alpha}=1
$$

so that the map simply exchanges the two terms of $\mathcal{R}_{q} \oplus \mathcal{R}_{q}$. On $\mathcal{R}_{q} \oplus \mathcal{R}_{q}$ we have the representation

$$
\theta_{R}^{1}=1
$$

If $d_{R}$ is to be a differential then the extension to higher order forms much be such that $d_{R}^{2}=0$. We have then

$$
\left(d_{R} x\right)^{2}=0
$$

It follows that

$$
d_{R} \theta_{R}^{1}=0, \quad\left(\theta_{R}^{1}\right)^{2}=0 .
$$

The module structure of $\Omega_{R}^{1}\left(\mathbb{R}_{q}^{1}\right)$ is given by the relations (4.10), which are equivalent to the condition that $\theta_{R}^{1}$ commute with all the elements of $\mathbb{R}_{q}^{1}$. The algebraic structure of $\Omega_{R}^{*}\left(\mathbb{R}_{q}^{1}\right)$ is defined by the relations (4.14).

The algebra $\mathbb{R}_{q}^{1}$ is a subalgebra of the graded algebra of forms $\Omega^{*}\left(\mathbb{R}_{q}^{1}\right)$ and the representation (2.2) can be extended to a representation of the latter. Again since $\Omega_{R}^{1}\left(\mathbb{R}_{q}^{1}\right)$ is a free $\mathbb{R}_{q}^{1}$-module of rank one with the special basis $\theta_{R}^{1}$ we can identify

$$
\Omega_{R}^{*}\left(\mathbb{R}_{q}^{1}\right)=\bigwedge^{*} \otimes \mathbb{R}_{q}^{1}
$$

where $\bigwedge^{*}$ is the exterior algebra over $\mathbb{C}^{1}$ and so the extension is trivial. The $\theta_{R}^{1}$ here is to be interpreted as an element on the $\Lambda^{*}$ and the equality gives its representation 
as the unit in $\mathbb{R}_{q}^{1}$. The second of Equations 4.14 is to be interpreted then as the equation $1 \wedge 1=0$ in the exterior algebra.

The forms $\theta^{1}, \bar{\theta}^{1}$ and $\theta_{R}^{1}$ are closed. They are also exact. In fact if we define $K \in \mathbb{R}_{q}^{1} \times \mathbb{R}_{q}^{1}$ by

$$
K=z\left(\Lambda^{-1}, \Lambda\right), \quad K^{*}=K,
$$

then we find that

$$
\theta^{1}=d\left(z \Lambda^{-1} y\right), \quad \bar{\theta}^{1}=\bar{d}(z \Lambda y), \quad \theta_{R}^{1}=d_{R}(K y) .
$$

One can always write a number $x$ as the sum of a complex number $z$ and its complex conjugate $\bar{z}$. If some invariance property were to forbid us from writing any formula involving $d x$ then we would have to express it in terms of $d z$ and $d \bar{z}$. What we have done in this section is equivalent to just this. It is not even interesting from the point of view of module structure; we have considered the simple direct sum of two free modules and the submodule defined in (4.5) is also free, with $\theta_{R}^{1}$ as a generator. To a certain extent what we have done is similar in spirit to the doubling of the rank of the module of 1-forms proposed by previous authors accompanied by an 'abstract' isomorphism [12] to then effectively reduce the rank by one half. One can also construct a (smaller) real differential calculus over $\mathbb{R}_{q}^{1}$ using the derivation ad $\left(\lambda_{1}+\bar{\lambda}_{1}\right)$ but this calculus has a set of 1 -forms with a more complicated module structure than the one we have constructed. There would be no simple relation like (4.10) between $x d_{R} x$ and $d_{R} x x$.

The limit $q \rightarrow 1$ is rather difficult to control. From the relations of the algebra and the two differential calculi one might expect $\Lambda \rightarrow 1$. This is consistent with the limiting relations $e_{1} x=\bar{e}_{1} x=x$ and the intuitive idea that $x$ is an exponential function on the line. However the representation (2.2) of the algebra becomes quite singular. In the representation one has rather $x \rightarrow 1$. This would imply that the parameters $\alpha$ and $\bar{\alpha}$ must tend to zero as $q \rightarrow 1$. If one renormalizes according to (2.5) then one finds that the relation (2.4) is consistent with the limit $\Lambda \rightarrow 1$ as $q \rightarrow 1$. We shall assume this to be the case. We have then

$$
\lim _{q \rightarrow 1} \mathcal{A}_{R}=\mathcal{A}
$$

and the real differential calculus coincides with the diagonal elements of the product in (4.1).

\section{The $q$-deformed derivatives}

We now look at the differential calculus from the dual point of view. We introduce a twisted derivation $\partial_{1}$ dual to the differential $d$. For every $f \in \mathbb{R}_{q}^{1}$ we require that $d f\left(\partial_{1}\right)=\partial_{1} f$. If one uses the (historical) convention of writing $d f=d x f_{1}$, with the differential to the left, then this means that for arbitrary $f \in \mathbb{R}_{q}^{1}$

$$
\partial_{1} f=d x\left(\partial_{1}\right) f_{1}=f_{1} .
$$

Consider the case $f=x^{2}$. Then $d f=d x(1+q) x$ and so $f_{1}=(1+q) x$. But $d f\left(\partial_{1}\right)=$ $\partial_{1} x^{2}$. Therefore $\partial_{1} x^{2}=(1+q) x$ Consider the case $f=\Lambda x$. Then $d f=d x q^{-1} \Lambda$ and so $f_{1}=q^{-1} \Lambda$. But $d f\left(\partial_{1}\right)=\partial_{1}(\Lambda x)$. Therefore $\partial_{1}(\Lambda x)=q^{-1} \Lambda$. By considering arbitrary polynomials in $x$ and $\Lambda$ one finds the commutation relations

$$
\partial_{1} x=1+q x \partial_{1}, \quad \partial_{1} \Lambda=q^{-1} \Lambda \partial_{1} .
$$


It is to be noticed here that the module structure of the differential forms is considered as fixed and the commutation relations above are derived from it. When a differential calculus is based on derivations the module structure of the forms is derived from the Leibniz rule. Notice also that we are here considering $x$ as an operator on $\mathbb{R}_{q}^{1}$ considered as a vector space. We should in principle put a hat on it to distinguish it from the element $x$ in $\mathbb{R}_{q}^{1}$ considered as an algebra. We are also considering $\partial_{1}$ as an operator on $\mathbb{R}_{q}^{1}$ considered as a vector space. We should put a hat on it also to distinguish it from the twisted derivation of $\mathbb{R}_{q}^{1}$. We have effectively enlarged the algebra $\mathbb{R}_{q}^{1}$ to an algebra $T_{q} \mathbb{R}_{q}^{1}$ by adding to it the element $\partial_{1}$ with the commutation relations (5.2).

We noticed above that the differential $d x$ was not real. In general $(d f)^{*} \neq d f^{*}$. Closely related to this is the fact that the derivation $\partial_{1}$ is not real. In general $\left(\partial_{1} f\right)^{*} \neq$ $\partial_{1} f^{*}$. Therefore $\partial_{1}$ is not antihermitian considered as an element of $T_{q} \mathbb{R}_{q}^{1}$. One can introduce a second twisted derivation $\bar{\partial}_{1}$ dual to the differential $\bar{d}$. It is defined by the commutation relations

$$
\bar{\partial}_{1} x=1+q^{-1} x \bar{\partial}_{1}, \quad \bar{\partial}_{1} \Lambda=q^{-1} \Lambda \bar{\partial}_{1} .
$$

We have then a second extension $\bar{T}_{q} \mathbb{R}_{q}^{1}$ of $\mathbb{R}_{q}^{1}$.

The representation $(2.2)$ of $\mathbb{R}_{q}^{1}$ can be extended to a representation of $T_{q} \mathbb{R}_{q}^{1}$ and $\bar{T}_{q} \mathbb{R}_{q}^{1}$. We have respectively 25, 15.

$$
\begin{aligned}
& \partial_{1}|k\rangle=-z^{-1} q^{-k-1}|k\rangle+z^{-1} \beta q^{-k}|k-1\rangle, \\
& \bar{\partial}_{1}|k\rangle=z^{-1} q^{-k}|k\rangle+z^{-1} \bar{\beta} q^{-k-1}|k+1\rangle
\end{aligned}
$$

with again two arbitrary complex parameters $\beta$ and $\bar{\beta}$. It is easy to see that the commutation relations (5.2) and (5.3) are satisfied. Again the representations are certainly not unique. We shall conclude from (5.4) that

$$
\bar{T}_{q} \mathbb{R}_{q}^{1}=T_{q} \mathbb{R}_{q}^{1}
$$

Due to the presence of the unit on the right-hand side of the commutation relations (5.2) and (5.3) the relation between $\partial_{1}^{*}$ and $\bar{\partial}_{1}$ is not as simple as it was in the case of the differentials. Adding the adjoint of Equations (5.2) to Equations (5.3) yields the commutation relations

$$
x\left(q \partial_{1}^{*}+\bar{\partial}_{1}\right)=q\left(q \partial_{1}^{*}+\bar{\partial}_{1}\right) x, \quad \Lambda\left(q \partial_{1}^{*}+\bar{\partial}_{1}\right)=q\left(q \partial_{1}^{*}+\bar{\partial}_{1}\right) \Lambda .
$$

From this we can conclude that

$$
q \partial_{1}^{*}+\bar{\partial}_{1}=c_{1} \Lambda x^{-1}
$$

for some constant $c_{1}$. In terms of the parameters of the representation (5.4) we find the expression

$$
c_{1}=z^{-1}\left(\beta^{*}+q^{-1} \bar{\beta}\right)
$$

for $c_{1}$.

We can consider the derivations $e_{1}$ and $\bar{e}_{1}$ also as elements of $T_{q} \mathbb{R}_{q}^{1}$. As such they satisfy the commutation relations

$$
\begin{array}{ll}
e_{1} x=q \Lambda x+x e_{1}, & e_{1} \Lambda=\Lambda e_{1}, \\
\bar{e}_{1} x=\Lambda^{-1} x+x \bar{e}_{1}, & \bar{e}_{1} \Lambda=\Lambda \bar{e}_{1} .
\end{array}
$$

These are the analogs of $(5.2)$ and (5.3) respectively. One sees immediately that as elements of $T_{q} \mathbb{R}_{q}^{1}$ the $e_{1}$ and $\bar{e}_{1}$ satisfy the relation

$$
e_{1}^{*}+\bar{e}_{1}=c_{2}
$$


for some constant $c_{2}$. This is the derivation analog of (5.5). It is to be compared with (3.3). The equation $e_{1} f=\left[\lambda_{1}, f\right]$ relates the derivation $e_{1}$ to the operator $\lambda_{1}$. There is an ambiguity

$$
\lambda_{1} \mapsto \lambda_{1}+z^{-1} \gamma
$$

in this identification which depends on a complex parameter $\gamma$. A similar ambiguity exists for $\bar{\lambda}_{1}$. As operators on $\mathcal{R}_{q}$ we find that we can write then

$$
e_{1}=z^{-1} \gamma+\lambda_{1}, \quad \bar{e}_{1}=z^{-1} \bar{\gamma}+\bar{\lambda}_{1}
$$

and in terms of $\gamma$ and $\bar{\gamma}$ we find the expression

$$
c_{2}=z^{-1}\left(\gamma^{*}+\bar{\gamma}\right)
$$

for $c_{2}$.

If we use the expressions (3.5) then by comparing (5.2) and (5.3) with (5.6) we deduce as above the relations

$$
\partial_{1}-\Lambda^{-1} x^{-1} e_{1}=c_{3} \Lambda^{-1} x^{-1}, \quad \bar{\partial}_{1}-q^{-1} \Lambda x^{-1} \bar{e}_{1}=c_{4} \Lambda x^{-1},
$$

where $c_{3}$ and $c_{4}$ are two arbitrary constants. We shall here set these two constants equal to zero. This means that we choose

$$
\gamma=\beta, \quad \bar{\gamma}=\bar{\beta}
$$

We find then the relations

$$
\partial_{1}=\theta_{1}^{1} e_{1}=\Lambda^{-1} x^{-1} e_{1}, \quad \bar{\partial}_{1}=\bar{\theta}_{1}^{1} \bar{e}_{1}=q^{-1} \Lambda x^{-1} \bar{e}_{1}
$$

between the derivations $\left(e_{1}, \bar{e}_{1}\right)$ and the twisted derivations $\left(\partial_{1}, \bar{\partial}_{1}\right)$. We recall that the vector space $\operatorname{Der}\left(\mathbb{R}_{q}^{1}\right)$ is not a left module over the algebra $\mathbb{R}_{q}^{1}$. As operators on $\mathcal{R}_{q}$ one finds the representations

$$
e_{1}|k\rangle=-z^{-1}|k+1\rangle+z^{-1} \beta|k\rangle, \quad \bar{e}_{1}|k\rangle=z^{-1}|k-1\rangle+z^{-1} \bar{\beta}|k\rangle
$$

for the derivations. It follows directly from (5.8) that

$$
\beta \Lambda^{-1}=1+q z x \partial_{1}, \quad \bar{\beta} \Lambda=-1+z x \bar{\partial}_{1} .
$$

Inverting these expressions, we find that in fact

$$
\bar{T}_{q} \mathbb{R}_{q}^{1}=T_{q} \mathbb{R}_{q}^{1}=\mathbb{R}_{q}^{1} .
$$

Using the adjoint of the Equations (5.2) we can write (5.11) in the form

$$
x \partial_{1}^{*}-1=\Lambda e_{1}^{*}, \quad x \bar{\partial}_{1}=\Lambda \bar{e}_{1},
$$

from which using (5.7) we deduce that

$$
x\left(\partial_{1}^{*}+\bar{\partial}_{1}\right)=1+z^{-1}(\beta+\bar{\beta}) \Lambda .
$$

It is interesting to note that $d \partial_{1}$ and $d \bar{\partial}_{1}$ are well-defined and not equal to zero.

From (4.9) we are prompted to introduce the antihermitian element $e_{R 1}$ of $T_{q} \mathbb{R}_{q}^{1}$ with the commutation relations

$$
\left[e_{R 1}, x\right]=\left(q \Lambda, \Lambda^{-1}\right) x, \quad\left[e_{R 1}, \Lambda\right]=0 .
$$


From the definition of $e_{R 1}$ as derivation one sees that the solution is given by

$$
e_{R 1}=\lambda_{R 1}+c_{R}
$$

for some complex parameter $c_{R}$. If $\beta= \pm 1, \bar{\beta}=\mp 1$ then

$$
c_{R}= \pm z^{-1}(1,-1)
$$

and using (5.12) one finds the relation

$$
e_{R 1}= \pm\left(x \bar{\partial}_{1}, q x \partial_{1}\right)
$$

between $e_{R 1}$ on the one hand and $x \partial_{1}$ and $x \bar{\partial}_{1}$ on the other.

It does not seem to be possible to construct a real metric on the twisted derivations without ambiguity. The problem is complicated by the fact that, whereas $\bar{d} x=(d x)^{*}$, from (5.13) one sees that $\bar{\partial}_{1} \neq-\partial_{1}^{*}$. It would be natural to define, for example, $\partial_{R 1}$ by the condition

$$
d_{R} x\left(\partial_{R 1}\right)=1 .
$$

However it is easy to see that this is not possible since if $\partial_{R 1}$ is to be antihermitian as an operator then $\partial_{R 1} x$ cannot be hermitian as an element of the algebra and so cannot be set equal to one. One could make the choice [11]

$$
\partial_{R 1}=\frac{1}{2}\left(\partial_{1}-\partial_{1}^{*}\right)
$$

or the choice

$$
\bar{\partial}_{R 1}=\frac{1}{2}\left(\bar{\partial}_{1}^{*}-\bar{\partial}_{1}\right)
$$

or any combination of the two. We find from the representation (5.4) that

$$
x \partial_{R 1}=\frac{1}{2} z^{-1} \beta\left(q^{-1} \Lambda^{-1}-\Lambda\right), \quad \partial_{R 1} x=\frac{1}{2} z^{-1} \beta\left(\Lambda^{-1}-q^{-1} \Lambda\right)
$$

from which we conclude that

$$
q \partial_{R 1} x=\frac{1}{2}(q+1) \beta \Lambda^{-1}+x \partial_{R 1}, \quad \Lambda \partial_{R 1}=q \partial_{R 1} \Lambda .
$$

This is to be compared with (5.2) and (5.3). In particular we find that as twisted derivation

$$
d_{R} x\left(\partial_{R 1}\right)=\partial_{R 1} x=\frac{1}{2 q}(q+1) \beta \Lambda^{-1} \neq 1 .
$$

In view of this ambiguity we shall use the derivation $e_{R 1}$ to define hermitian differential operators.

\section{Integration}

Because of (3.6), (4.11) and (4.12) we define [5] the (definite) integrals to be the linear maps from $\Omega_{q}^{1}\left(\mathbb{R}_{q}^{1}\right), \bar{\Omega}_{q}^{1}\left(\mathbb{R}_{q}^{1}\right)$ and $\Omega_{R}^{1}\left(\mathbb{R}_{q}^{1}\right)$ into the complex numbers given by respectively

$$
\int f_{1} \theta^{1}=\operatorname{Tr}\left(f_{1}\right), \quad \int \bar{f}_{1} \bar{\theta}^{1}=\operatorname{Tr}\left(\bar{f}_{1}\right), \quad \int f_{R} \theta_{R}^{1}=\operatorname{Tr}\left(f_{R}\right) .
$$

In the last expression the trace includes the sum of the components. Since the 'space' is 'noncompact' we have

$$
\int \theta^{1}=\operatorname{Tr}(1)=\infty
$$


In all cases the integral of an exact form is equal to zero. For example

$$
\int d f=\int e_{1} \theta^{1}=\operatorname{Tr}\left(\left[\lambda_{1}, f\right]\right)=0 .
$$

This is in fact rather formal since it is possible for the commutator of two unbounded operators to have a non-vanishing trace. The integral

$$
\int d x=\operatorname{Tr}\left(e_{1} x\right)=q \operatorname{Tr}(\Lambda x)=0
$$

but the integral

$$
\int d\left(\Lambda^{-1} x\right)=\int \Lambda^{-1} d x=q \operatorname{Tr}(x)=\sum_{-\infty}^{\infty} q^{k}=\infty .
$$

We can interpret the trace as an inner-product on the algebra by setting

$$
\langle f \mid g\rangle \equiv \int\left(f^{*} g\right) \theta^{1}=\operatorname{Tr}\left(f^{*} g\right) .
$$

The trace defines a state which characterizes the representation we are using. It follows immediately from the definition that an operator which is hermitian as an element of the algebra is also hermitian with respect to the inner-product.

\section{The geometry}

It is now possible to give an intuitive interpretation of the metric (1.2) in terms of observables. One can think of the algebra $\mathbb{R}_{q}^{1}$ as describing a set of 'lines' $x$ embedded in a 'plane' $(x, \Lambda)$ and defined by the condition ' $\Lambda=$ constant'. To within a normalization the unique metric is given by

$$
g\left(\theta_{R}^{1} \otimes \theta_{R}^{1}\right)=1 .
$$

Using it we introduce the element

$$
g^{\prime 11}=g\left(d_{R} x \otimes d_{R} x\right)=\left(e_{R 1} x\right)^{2} g\left(\theta_{R}^{1} \otimes \theta_{R}^{1}\right)=\left(e_{R 1} x\right)^{2}
$$

of the algebra. Then

$$
\sqrt{g^{\prime 11}}=e_{R 1} x, \quad\left(\sqrt{g^{\prime 11}}\right)^{*}=\sqrt{g^{\prime 11}} .
$$

We have a representation of $x$ and $d_{R} x$ on the Hilbert space $\mathcal{R}_{q}$. In this representation the distance $s$ along the 'line' $x$ is given by the expression

$$
d s(k)=\| \sqrt{g_{11}^{\prime}} d_{R} x(|k\rangle+\overline{|k\rangle}) \|
$$

with as usual $g_{11}^{\prime}=\left(g^{\prime 11}\right)^{-1}$. This comes directly from the original definition of $d x$ as an 'infinitesimal displacement'. Using (4.12) we find that

$$
d s(k)=\||k\rangle+\overline{|k\rangle} \|=1 .
$$

The 'space' is discrete [27] and the spacing between 'points' is uniform. The distance operator $s$ can be identified with the element $y$ introduced in (2.3). This means that if we measure $y$ using laboratory units, introduced in Equation (2.5) then we shall do 
the same with $s$. In these units then the distance between neighboring 'points' is given by

$$
d s(k)=z .
$$

If one forgets the reality condition then one can introduce the hermitian metric $g$ with $g\left(\bar{\theta}^{1} \otimes \theta^{1}\right)=1$. One finds then

$$
g^{\prime 11}=g(\bar{d} x \otimes d x)=\bar{e}_{1} x e_{1} x=q^{2} x^{2}
$$

and one concludes that

$$
d s(i)=\| \sqrt{g_{11}^{\prime}} d x|k\rangle \|=q .
$$

One can also introduce the hermitian metric $g$ with $g\left(e_{1} \otimes e_{1}^{*}\right)=1$. One finds then

$$
g_{11}^{\prime}=g\left(\partial_{1} \otimes \partial_{1}^{*}\right)=\Lambda^{-1} x^{-1} g\left(e_{1} \otimes e_{1}^{*}\right) x^{-1} \Lambda=q^{-2} x^{-2}
$$

and one finds again the expression (7.6) for the distance.

If one neglects also hermiticity and introduces a metric $g$ with $g\left(\theta^{1} \otimes \theta^{1}\right)=1$ then one finds that

$$
g^{\prime 11}=g(d x \otimes d x)=\left(e_{1} x\right)^{2}=(q \Lambda x)^{2} .
$$

Since we have defined a 'tangent space' $T_{q} \mathbb{R}_{q}^{1}$ and a 'cotangent space' $\Omega^{1}\left(\mathbb{R}_{q}^{1}\right)$ it is of interest to interpret the metric as a map

$$
\Omega_{R}^{1}\left(\mathbb{R}_{q}^{1}\right) \stackrel{g}{\longrightarrow} T_{q} \mathbb{R}_{q}^{1}
$$

This corresponds to the 'raising of indices' in ordinary geometry. As such it can be defined as the map $g\left(\theta^{1}\right)=e_{1}$. A short calculation yields that this is equivalent to

$$
g(d x)=g^{\prime 11} \partial_{1}
$$

as it should be. Although both $d x$ and $\partial_{1}$ have been represented on the same Hilbert space we cannot conclude that in this representation the map (7.8) is given by $g=1$. That is, as operators on $\mathcal{R}_{q}$, we have $d x \neq g^{\prime 11} \partial_{1}$. One finds in fact that

$$
\left(d x-g^{\prime 11} \partial_{1}\right)|k\rangle=q^{2} z^{-1} q^{2 k}|k+2\rangle+\left(\alpha-\beta q z^{-1}\right) q^{k}|k+1\rangle .
$$

We define covariant derivatives $D$ and $\bar{D}$ on $\Omega^{1}\left(\mathbb{R}_{q}^{1}\right)$ as maps

$$
\Omega^{1}\left(\mathbb{R}_{q}^{1}\right) \stackrel{D}{\longrightarrow} \Omega^{1}\left(\mathbb{R}_{q}^{1}\right) \otimes \Omega^{1}\left(\mathbb{R}_{q}^{1}\right), \quad \bar{\Omega}^{1}\left(\mathbb{R}_{q}^{1}\right) \stackrel{\bar{D}}{\longrightarrow} \bar{\Omega}^{1}\left(\mathbb{R}_{q}^{1}\right) \otimes \bar{\Omega}^{1}\left(\mathbb{R}_{q}^{1}\right)
$$

which satisfy [9] left and right Leibniz rules. The metric-compatible, torsion-free connections are given by the covariant derivatives

$$
D \theta^{1}=0, \quad \bar{D} \bar{\theta}^{1}=0 .
$$

These equations can be written also as

$$
D(d x)=q^{2} \Lambda^{2} x \theta^{1} \otimes \theta^{1}, \quad \bar{D}(\bar{d} x)=\Lambda^{-2} x \bar{\theta}^{1} \otimes \bar{\theta}^{1} .
$$

The real torsion-free covariant derivative compatible with the real metric is given by

$$
D_{R} \theta_{R}^{1}=0
$$

This can also be written in the form

$$
D_{R}\left(d_{R} x\right)=\left(q^{2} \Lambda^{2}, \Lambda^{-2}\right) x \theta_{R}^{1} \otimes \theta_{R}^{1} .
$$


The generalized flip $\sigma_{R}$ is given 8 by $\sigma_{R}=1$. This yields [13 the involution

$$
\left(\theta_{R}^{1} \otimes \theta_{R}^{1}\right)^{*}=\theta_{R}^{1} \otimes \theta_{R}^{1}
$$

on the tensor product if the covariant derivative (7.9) is to be real:

$$
D_{R} \xi^{*}=\left(D_{R} \xi\right)^{*}
$$

The geometry is 'flat' in the sense that the curvature tensor defined by $D_{R}$ vanishes. The interpretation is somewhat unsatisfactory however here because of the existence of elements in the algebra which do not lie in the center but which have nevertheless vanishing exterior derivative. These elements play a relatively minor importance in the geometry of the algebras $\mathbb{R}_{q}^{n}$ for larger values of $n$ [24].

\section{Yang-Mills fields}

Consider the algebra $\mathcal{A}_{q}$ obtained by adding a time parameter $t \in \mathbb{R}$ to $\mathbb{R}_{q}^{1}: \mathcal{A}_{q}=$ $\mathcal{C}(\mathbb{R}) \otimes \mathbb{R}_{q}^{1}$. The tensor product is understood to include a completion with respect to the topologies. Choose $\mathcal{H}$ as the $\mathcal{A}_{q}$-bimodule which is free of rank $r$ as a left or right module and assume that is can be considered as an $\mathbb{R}_{q R}^{1}$-bimodule. Introduce a differential calculus over $\mathcal{A}_{q}$ by choosing the ordinary de Rham differential calculus over the time parameter and $\Omega_{R}^{*}\left(\mathbb{R}_{q}^{1}\right)$ over the factor $\mathbb{R}_{q}^{1}$. One defines a covariant derivative of $\psi \in \mathcal{H}$ as a map

$$
\mathcal{H} \stackrel{D}{\longrightarrow} \Omega^{1}\left(\mathcal{A}_{q}\right) \otimes \mathcal{H}
$$

which satisfies the left Leibniz rule

$$
D(f \psi)=d f \otimes \psi+f D \psi .
$$

We shall henceforth drop the tensor product symbol and write

$$
D \psi=d t D_{t} \psi+D_{R} \psi
$$

We define

$$
D_{t} \psi=\left(\partial_{t}+A_{t}\right) \psi
$$

Since $\mathcal{A}_{q}$ is an algebra with involution we can choose as gauge group the set $\mathcal{U}_{q}(r)$ of unitary elements of $M_{r}\left(\mathcal{A}_{q}\right)$. A gauge transformation $g \in \mathcal{U}_{q}$ is a map

$$
\psi \mapsto \psi g, \quad A \mapsto g^{-1} A g+g^{-1} d g
$$

which is independent of $\Lambda$. It is easy to see [7, 21] that $\phi_{R}=A_{R}-\theta_{R}$ transforms under the adjoint action of the gauge group. We define then

$$
D_{R} \psi=-\theta_{R} \psi-\psi \phi_{R}
$$

This covariant derivative is covariant under the right action of the gauge group and satisfies a left Leibniz rule. The covariant derivative and the field strength transform as usual

$$
D \psi \mapsto(D \psi) g, \quad F \mapsto g^{-1} F g
$$

One can also write $D_{R} \psi=\theta^{1} D_{R 1} \psi$ with $D_{R 1}=e_{R 1}+A_{R 1}$. The field strength can be written then

$$
F \psi=D^{2} \psi=d t \theta_{R}^{1} \psi F_{t 1}
$$


with

$$
F_{t 1}=\partial_{t} A_{R 1}-e_{R 1} A_{t}
$$

When the gauge potential vanishes one has from (8.1)

$$
D_{R} \psi=\theta_{R}^{1} e_{R 1} \psi
$$

To form invariants we introduce the metric (7.1). We define the matter action $S_{M}$ by analogy with the commutative case:

$$
S_{M}=\operatorname{Tr} \int d t\left(D_{t} \psi^{*} D_{t} \psi+D_{R 1} \psi^{*} D_{R 1} \psi\right)
$$

The trace is here over the Lie algebra of the gauge group and over the representation of the algebra $\mathbb{R}_{q}^{1}$. We define also as usual the Yang-Mills action $S_{Y M}$ as

$$
S_{Y M}=\frac{1}{4} \operatorname{Tr}\left(F_{t 1} F_{t 1}\right)
$$

and the action to be the sum $S=S_{M}+S_{Y M}$. The trace however would depend on the representation of the algebra and it is not obvious how one should vary $S$. To define the trace we must consider explicitly the representations of $\psi$ and $A_{t}$ and $A_{R 1}$ on the Hilbert space $\mathcal{R}_{q}$. Since 'space' has only one dimension there are no dynamical solutions to the vacuum Yank-Mills equations. There is no dispersion relation since there are no transverse modes. One can also write the action as an integral using the definition of Section 6 .

In the spirit of noncommutative geometry the 'state vectors' play the role of the set of points. The eigenvalues of an observable of the algebra, in a given representation, are the noncommutative equivalents of the values which its classical counterpart can take. An eigenvector associated to a given eigenvalue describes a set of states in which the given observable can take the prescribed value. This is exactly like quantum mechanics but in position space. Consider now a field configuration, for example an element of the initial algebra $\mathcal{A}$ if it is a scalar field or an element of an algebra of forms over $\mathcal{A}$ if it is a Yang-Mills field. Suppose that both of these algebras have a representation on some Hilbert space and suppose that there exists a well-defined energy functional which is also represented as an operator on the Hilbert space. A vacuum configuration would be then an element of the algebra which is such that the expectation value of the corresponding value of the energy functional in any state vanishes. This is the same as saying that a field is equal to zero if the value of its energy is equal to zero at every point of space.

In the 'classical' noncommutative case a derivation, if it exists at all, is a map of the algebra into itself; it is not an element of the algebra. In the case we are considering this is not so. The algebra $\mathbb{R}_{q}^{1}$ is a position space described by the subalgebra generated by $x$ extended by $\Lambda$ which is an element of the associated phase space. The differential calculus however is somehow restricted to the position space by the condition $d \Lambda=$ 0. Both the initial algebra and the algebra of forms contain then operators which correspond to derivations. We have in fact given the representation of these elements on $\mathcal{R}_{q}$, the same Hilbert space on which the 'position' variables and the forms are represented. A vacuum configuration is then something different than it is in the 'classical' case.

Consider, for example, a scalar field $\psi(x) \in \mathbb{R}_{q}^{1}$ and suppose that the energy functional is of the simple form $\mathcal{E}=\left(e_{R 1} \psi\right)^{*}\left(e_{R 1} \psi\right)$. If $e_{R 1}$ is considered as partial derivative then $\mathcal{E}=\mathcal{E}(x)$ depends on the position variable alone and a vacuum configuration would 
be one in which the expectation value of $\mathcal{E}$ vanishes for all state vectors. This would normally be one with $\psi=\psi_{0}$ for some $\psi_{0}$ ith $e_{R 1} \psi_{0}=0$. However $e_{R 1}$ as operator belongs also to $\mathbb{R}_{q}^{1}$ and the expression for the energy functional could be interpreted as one quadratic in this element. In this case the only possible vacuum configuration would be $\psi=0$. There exist particular state vectors for which the energy functional of more complicated configurations vanish. As an example of this we return to the Yang-Mills case. One would like a vacuum to be given as usual by $\psi=1$ (the unit cyclic vector of $\mathcal{H}$ ) and $A_{R}=0$. One finds then as condition that

$$
D_{R} \psi(|k\rangle+\overline{|k\rangle})=d_{R}(|k\rangle+\overline{|k\rangle})=0 .
$$

To be concrete we shall suppose that $c_{R}$ is given by (5.15). From (4.8) one sees that the vacuum equation leads to the conditions

$$
(\Lambda-1) \sum_{k} a_{k}|k\rangle=0, \quad\left(\Lambda^{-1}-1\right) \sum_{k} \bar{a}_{k}|k\rangle=0
$$

on the two copies of $\mathcal{R}_{q}$. The vacuum state vectors form then a subspace of $\mathcal{R}_{q}$ of dimension 2 spanned by the vectors given by $a_{k}=1, \bar{a}_{k}=1$. These values depend of course on our choice of $c_{R}$. All vacuum state vectors have infinite norm. The vacuum state vectors would be the analog of the vacuum of quantum field theory which is defined as the vector in Fock space which is annihilated by the energy-momentum vector of Minkowski space. The Fock-space vector is taken to be of unit norm.

\section{The Schrödinger equation}

Recall that on a curved manifold with metric $g_{\mu \nu}$ the laplacian is defined to be the hermitian operator

$$
\Delta=-g^{\mu \nu} D_{\mu} D_{\nu}=-\frac{1}{\sqrt{g}} \partial_{\mu}\left(\sqrt{g} g^{\mu \nu} \partial_{\nu}\right)
$$

Because of (8.1) on the geometry defined by $\Omega_{R}^{*}\left(\mathbb{R}_{q}^{1}\right)$, with metric (7.1), this becomes

$$
\Delta_{R} \psi=-e_{R 1}^{2} \psi \text {. }
$$

We shall suppose that the gauge-covariant Schrödinger equation has the usual form

$$
i D_{t} \psi=\frac{1}{2 m} \Delta_{R} \psi
$$

where $\Delta_{R}$ is the Laplace operator (9.1). There is a conserved current which we write in the form

$$
\partial_{t} \rho=D_{R 1} J_{R}^{1}
$$

with as usual

$$
\rho=\psi^{*} \psi, \quad J_{R 1}=\frac{i}{2 m}\left(\psi^{*} D_{R 1} \psi-D_{R 1} \psi^{*} \psi\right) .
$$

The conservation law follows directly from the field equations. Normally one derives the latter from an action principle. In the present situation this would be a non-relativistic form of the expression (8.2):

$$
S=\operatorname{Tr} \int d t\left(i \psi^{*} D_{t} \psi-\frac{1}{2 m} D_{R 1} \psi^{*} D_{R 1} \psi\right) .
$$


Consider the relativistic case and assume the usual form

$$
--\partial_{t}^{2} \psi=\Delta_{R} \psi+m^{2} \psi
$$

for the Klein-Gordan equation. Suppose that $A_{R}=0$. The laplacian has then a set of 'almost' eigenvectors. From the commutation relations

$$
e^{i k y} \Lambda=e^{i k} \Lambda e^{i k y}
$$

one finds that

$$
e_{1} e^{i k y}=z^{-1}\left(e^{i k}-1\right) \Lambda e^{i k y}, \quad \bar{e}_{1} e^{i k y}=z^{-1}\left(1-e^{-i k}\right) \Lambda^{-1} e^{i k y}
$$

from which it follows that

$$
e_{R 1} e^{i k y}=i k L e^{i k y}
$$

where

$$
L=\frac{1}{2 i k z}\left(\left(e^{i k}-1\right) \Lambda,\left(1-e^{-i k}\right) \Lambda^{-1}\right) .
$$

From the expression (9.1) one concludes then that

$$
\Delta_{R} e^{i k y}=k^{2} L^{2} e^{i k y} .
$$

We could renormalize the space unit as in Equation (2.5) to laboratory units. If we keep the Planck units we must renormalize the time unit so it will be also in Planck units. We do this by the transformation

$$
z^{-1} t \mapsto t
$$

We find then that

$$
\psi=e^{-i \omega L t-k y)}
$$

is a solution to (9.4) provided the dispersion relation

$$
\left(\omega^{2}-k^{2}\right) z^{2} L^{2}=m^{2}
$$

is satisfied. However the above dispersion relations are misleading since $\omega$ can not be identified with the energy; the coefficient of the time coordinate is in fact the product $\omega L$ and we must set therefore

$$
E=\omega L
$$

We would like to consider $\psi$ as an element of a free $\mathcal{A}_{0}$-module. We recall that $\mathcal{A}_{0}$ is the commutative subalgebra of $\mathcal{A}$ generated by $x$. In general however $\mathcal{A}_{0}$ is not invariant under the action of the hermitian derivations. We consider then the limit $q \rightarrow 1$. In this limit we have argued that $\Lambda \rightarrow 1$ but at the same time $z \rightarrow 0$ so the following argument is subject to caution. We supposed that as $q \rightarrow 1$ we have $\bar{e}_{1} \rightarrow e_{1}$. In this rather singular limit we can identify then

$$
e_{R 1}=\frac{1}{2}\left(e_{1}+\bar{e}_{1}\right)(1,1)+o(z)
$$

and in this limit

$$
L=z^{-1} \frac{\sin k}{k}(1,1)+o(1) .
$$

This second equality seems to follow from the first but it is especially difficult to justify. If we accept it however then with the new time unit we find that

$$
E^{2}=\omega^{2} \frac{\sin ^{2} k}{k^{2}}
$$


and the dispersion relation in the relativistic case becomes

$$
E^{2}=m^{2}+\sin ^{2} k .
$$

If $k=\pi n$, with $n \in \mathbb{Z}$ then $E=0$ and one has

$$
e_{R 1} e^{-i k y}=0 .
$$

In the massless case this yields a set of 'stationary-wave' solutions to the field equations.

When $k<<\pi / 2$ (in Planck units) one obtains the usual dispersion relation $E^{2}=$ $m^{2}+k^{2}$. In the case $m<<1$ as $k \rightarrow \pi / 2$ then $E$ tends to a maximum value equal to 1 (again in Planck units). Values of $k$ greater than $\pi / 2$ would be difficult to interpret physically.. For comparison we recall that, neglecting the gap corrections, the dispersion relation for acoustical phonons on a lattice [17] is of the form

$$
E^{2}=\sin ^{2} \frac{k}{2}
$$

Here $E$ is the phonon energy and $k$ is the wave number. This has the same form as (9.5) when $m=0$. The factor $1 / 2$ is a convention. The first Brillouin zone is the range $-\pi \leq k \leq \pi$. There are also optical phonons which are similar to the case $m>0$ but they have a different dispersion relation. The 'space' $\mathbb{R}_{q}^{1}$ is not an ordinary crystal.

\section{Phase space}

If we wish to construct a real phase 'space' associated to the position 'space' we must define two hermitian operators which can play the role of 'position' and 'momentum'. We have already remarked that the distance operator $s$ can be identified with the element $y$ introduced in (2.3). As 'position' operator we choose then the renormalized $y$ given by (2.5). A short calculation shows that

$$
e_{R 1} y=z K^{-1}
$$

where the element $K$ was introduced in (4.15). If we consider then $e_{R 1}$ as an operator we have the commutation relation

$$
\left[e_{R 1}, y\right]=\left(\Lambda, \Lambda^{-1}\right)
$$

We have already noticed that $e_{R 1}$ is antihermitian. We define then the momentum associated to $y$ to be

$$
p_{y}=-i e_{R 1}=i z^{-1}\left(\Lambda,-\Lambda^{-1}\right) .
$$

We have not written the extra constant term $c_{R}$ of Equation (5.15) since it does not contribute to the commutation relation:

$$
\left[p_{y}, y\right]=-i h .
$$

We have here introduced the hermitian element

$$
h=\left(\Lambda, \Lambda^{-1}\right)
$$

of $\mathbb{R}_{q}^{1} \times \mathbb{R}_{q}^{1}$. Since we suppose that $\Lambda \rightarrow 1$ as $q \rightarrow 1$ we see that the commutation relation (10.1) becomes the ordinary one in this limit. 
We introduce the 'annihilation operator'

$$
a=\frac{1}{\sqrt{2}}\left(y+i p_{y}\right)
$$

Then from (10.1) follows the commutation relation

$$
\left[a, a^{*}\right]=h \text {. }
$$

It is not possible to express $h$ in terms of $a$ and $a^{*}$. The operator $e_{R 1}$ was taken as the antihermitian part of $e_{1}$; the operator $h$ depends also on the hermitian part. From (2.4) we find however that

$$
[a, h]=\frac{1}{2} z^{2}\left(a^{*}-a\right)
$$

To define a vacuum and a number operator we must 'dress' the operator $a$, introduce an operator $b$ so that the standard relations $\left[b, b^{*}\right]=1$ hold. It does not seem to be possible to do this exactly but it can be done as a perturbation series in $z$. One finds from (10.3) and (10.4) that

$$
b=h^{-1 / 2} a+\frac{1}{4} z^{2} a+\frac{1}{6} z^{2}\left(a-a^{*}\right)^{3}+o\left(z^{4}\right) .
$$

The vacuum is chosen then as usual by the condition $b|0\rangle=0$, the number operator is given by $N=b^{*} b$ and the number representation $|n\rangle$ for $n \in \mathbb{N}$ by

$$
|n\rangle=\frac{1}{\sqrt{n !}}\left(b^{*}\right)^{n}|0\rangle
$$

From (10.4) we find that

$$
[N, h]=\frac{1}{2} z^{2}\left(\left(b^{*}\right)^{2}-b^{2}\right)+o\left(z^{4}\right)
$$

There have been several $q$-deformed versions of the harmonic oscillator [20, 1, 4, 12, 19]. We shall reproduce here the equivalent calculations for the geometry described in Section 5. As hamiltonian we choose

$$
H=\frac{1}{2}\left(\Delta_{R}+y^{2}\right)
$$

in Planck units. This can be written also as $H=a^{*} a+\frac{1}{2} h$ and in terms of $b$ it is given by

$$
H=b^{*} h b+\frac{1}{2} h-\frac{1}{2} z^{2} b^{*} b+\frac{1}{6} z^{2}\left(\left(b-b^{*}\right)^{3} b-b^{*}\left(b-b^{*}\right)^{3}\right)+o\left(z^{4}\right) .
$$

We see then that in terms of the 'dressed' annihilation and creation operators the 'bare' hamiltonian is rather complicated. In particular the 'physical vacuum' is no longer an eigenvector of the 'bare' hamiltonian:

$$
H|0\rangle=\frac{1}{2}|0\rangle+\frac{1}{6} z^{2}|1\rangle+\frac{1}{\sqrt{2}} z^{2}|2\rangle-\frac{1}{\sqrt{6}} z^{2}|3\rangle+o\left(z^{4}\right) .
$$




\section{Non-local metrics}

We have devoted special attention to one particular metric on the calculus $\Omega_{R}^{*}\left(\mathbb{R}_{q}^{1}\right)$ for reasons given in Section 1: it is the only local metric. To test what this means in practice it is of interest to examine other metrics, which necessarily do not fulfill the locality condition. We would like the metric to have an associated linear connection so we shall first examine the most general form which this can take. We set as usual

$$
D_{1} \theta^{1}=-\omega^{1}{ }_{11} \theta^{1} \otimes \theta^{1}, \quad \bar{D}_{1} \bar{\theta}^{1}=--\bar{\omega}^{1}{ }_{11} \bar{\theta}^{1} \otimes \bar{\theta}^{1}
$$

as Ansatz for the linear connection. From the general theory [9] these must satisfy a left and right Leibniz rule

$$
\begin{array}{ll}
D_{1}\left(f \theta^{1}\right)=d f \otimes \theta^{1}-f \omega^{1}{ }_{11} \theta^{1} \otimes \theta^{1}, & D_{1}\left(\theta^{1} f\right)=\sigma\left(\theta^{1} \otimes d f\right)-\omega^{1}{ }_{11} f \theta^{1} \otimes \theta^{1} \\
\bar{D}_{1}\left(f \bar{\theta}^{1}\right)=\bar{d} f \otimes \bar{\theta}^{1}-f \bar{\omega}^{1}{ }_{11} \bar{\theta}^{1} \otimes \bar{\theta}^{1}, & \bar{D}_{1}\left(\bar{\theta}^{1} f\right)=\bar{\sigma}\left(\bar{\theta}^{1} \otimes \bar{d} f\right)-\bar{\omega}^{1}{ }_{11} f \bar{\theta}^{1} \otimes \bar{\theta}^{1}
\end{array}
$$

where $f \in \mathbb{R}_{q}^{1}$ and the generalized flips [8] $\sigma$ and $\bar{\sigma}$ can be written as

$$
\sigma\left(\theta^{1} \otimes \theta^{1}\right)=S \theta^{1} \otimes \theta^{1}, \quad \bar{\sigma}\left(\bar{\theta}^{1} \otimes \bar{\theta}^{1}\right)=\bar{S} \bar{\theta}^{1} \otimes \bar{\theta}^{1} .
$$

From the compatibility conditions

$$
\begin{array}{ll}
D_{1}\left(\Lambda \theta^{1}\right)=D_{1}\left(\theta^{1} \Lambda\right), & D_{1}\left(x \theta^{1}\right)=D_{1}\left(\theta^{1} x\right) \\
\bar{D}_{1}\left(\Lambda \bar{\theta}^{1}\right)=\bar{D}_{1}\left(\bar{\theta}^{1} \Lambda\right), & \bar{D}_{1}\left(x \bar{\theta}^{1}\right)=\bar{D}_{1}\left(\bar{\theta}^{1} x\right)
\end{array}
$$

it is easy to see that, to within a multiplicative constant, there are only two solutions, the one given previously in Section 6 and a new one given by

$$
\omega^{1}{ }_{11}=\Lambda, \quad S=q^{-1}, \quad \bar{\omega}_{11}^{1}=q \Lambda^{-1}, \quad \bar{S}=q .
$$

We set

$$
g\left(\theta^{1} \otimes \theta^{1}\right)=g^{11}, \quad g\left(\bar{\theta}^{1} \otimes \bar{\theta}^{1}\right)=\bar{g}^{11} .
$$

The metric compatibility condition [8] can be written

$$
d g^{11}=-(1+S) \omega^{1}{ }_{11} g^{11} \theta^{1}, \quad \bar{d} \bar{g}^{11}=-(1+\bar{S}) \bar{\omega}^{1}{ }_{11} \bar{g}^{11} \bar{\theta}^{1} .
$$

The possible solution to this equation, corresponding to the linear connection (11.1), is given by

$$
g_{11}=(q \Lambda x)^{2}, \quad \bar{g}_{11}=\left(\Lambda^{-1} x\right)^{2} .
$$

This can also be written in the form

$$
g(d x \otimes d x)=1, \quad g(\bar{d} x \otimes \bar{d} x)=1
$$

and the corresponding covariant derivative can be written also as

$$
D_{1} d x=0, \quad \bar{D}_{1} \bar{d} x=0 .
$$

The 'space' now is a discrete subset of the positive real axis with an accumulation point at the origin. The 'non-locality' means that if $f$ is a 'function' and $\alpha$ a form then the norm of $f \alpha$ cannot be equal to $f$ times the norm of $\alpha$. To see this we multiple (11.2) from the right by $x$. If we supposed that the metric were left and right linear then we would find

$$
x=x g(d x \otimes d x)=g(x d x \otimes d x)=q^{2} g(d x \otimes d x x)=q^{2} g(d x \otimes d x) x=q^{2} x .
$$


The first and fifth equalities are mathematical trivialities; the third follows directly from (3.1) . Therefore either the second or forth, or both, must be false. There are no 2 -forms and so the curvature and torsion of the non-local metric vanish.

The Ansatz for a covariant derivative on the real calculus is

$$
D_{R 1} \theta_{R}^{1}=--\omega_{R 11}^{1} \theta_{R}^{1} \otimes \theta_{R}^{1}
$$

If the generalized flip is given by $\sigma_{R}=\left(q^{-1}, q\right)$ then the appropriate involution 13 on the tensor product is given by

$$
\left(\theta_{R}^{1} \otimes \theta_{R}^{1}\right)^{*}=\left(q^{-1}, q\right)\left(\theta_{R}^{1} \otimes \theta_{R}^{1}\right)
$$

The solution to (11.3) is given by

$$
\omega_{R 11}^{1}=\left(\Lambda, q \Lambda^{-1}\right), \quad\left(\omega_{R 11}^{1}\right)^{*}=\left(q, q^{-1}\right) \omega_{R 11}^{1},
$$

The connection coefficient is not hermitian but the covariant derivative is real.

As an example of an application we return to the Yang-Mills fields written, for simplicity, using the derivations $D_{1}$ instead of $D_{R}$ and where now of course the $D_{1}$ must be chosen compatible with the new metric (11.2). The differential calculus over $\mathcal{A}_{q}$ is now the ordinary de Rham differential calculus over the time parameter and $\Omega^{*}\left(\mathbb{R}_{q}^{1}\right)$ over the factor $\mathbb{R}_{q}^{1}$. Otherwise all is as before in Section 8 except that $A$ is now given by

$$
A=d t A_{t}+\theta^{1} A_{1}
$$

and

$$
d \psi=d t \partial_{t} \psi+\theta^{1} e_{1} \psi
$$

with $e_{1} \psi=\left[\lambda_{1}, \psi\right]$. More important, the action $S$ becomes

$$
S_{M}=\operatorname{Tr}\left(D_{t} \psi^{*} D_{t} \psi\right)+g^{11} \operatorname{Tr}\left(D_{1} \psi^{*} D_{1} \psi\right)+\frac{1}{4} g^{11} \operatorname{Tr}\left(F_{t 1} F_{t 1}\right) .
$$

The metric coefficient $g^{11}=(q \Lambda x)^{-2}$ does not commute with the other factors in this expression so there is an ordering ambiguity. But it must be outside the trace in order not to destroy gauge invariance. Motivated by Equation (5.11) we introduce the 'twisted' covariant derivative $\nabla_{1}$ by the equation

$$
D_{1}=\sqrt{g_{11}} \nabla_{1} .
$$

If we write also $A_{1}=\sqrt{g_{11}} B_{1}$ and set $D_{t}=\nabla_{t}$ and $G_{t 1}=\partial_{t} B_{1}-\partial_{1} B_{t}$ then we find that

$$
\nabla=\partial_{1}+B_{1}, \quad F_{t 1}=\sqrt{g_{11}} G_{t 1}
$$

and we can write the action in the form

$$
S_{M}=\operatorname{Tr}\left(\nabla_{t} \psi^{*} \nabla_{t} \psi\right)+g^{11} \operatorname{Tr}\left(\sqrt{g_{11}} \nabla_{1} \psi^{*} \sqrt{g_{11}} \nabla_{1} \psi\right)+\frac{1}{4} g^{11} \operatorname{Tr}\left(\sqrt{g_{11}} G_{t 1} \sqrt{g_{11}} G_{t 1}\right) .
$$

We identify $\psi$ with an element $\psi(x)$ in the subalgebra of $\mathbb{R}_{q}^{1}$ generated by the element $x$ and we suppose also that $B_{1}=B_{1}(x)$. The action can be written then in the form

$$
S_{M}=\operatorname{Tr}\left(\nabla_{t} \psi^{*} \nabla_{t} \psi\right)+\Lambda^{-1} \operatorname{Tr}\left(\nabla_{1} \psi^{*} \Lambda \nabla_{1} \psi\right)+\frac{1}{4} \Lambda^{-1} \operatorname{Tr}\left(G_{t 1} \Lambda G_{t 1}\right) .
$$

It is the quantity $\Lambda G_{t 1}$ which is gauge covariant. 
As a second example of we return to the Schrödinger equation, written again using the covariant derivative $D_{1}$ compatible with the metric (11.2). There are two possible forms for the Laplace operator $\Delta$. In the absence of a gauge potential one can choose either

$$
\Delta=-g^{11} D_{1} D_{1}=--q \Lambda^{-2} x^{-2} e_{1}^{2}+q \Lambda^{-1} x^{-2} e_{1}
$$

or

$$
\Delta=-\frac{1}{\sqrt{g}}\left(e_{1} \sqrt{g} g^{11} e_{1}\right)=--q \Lambda^{-2} x^{-2} e_{1}^{2}+\Lambda^{-1} x^{-2} e_{1} .
$$

The two coincide when $q=1$. We shall choose the latter. If we introduce then the current 'density'

$$
\sqrt{g} J^{1}=\frac{i}{2 m} \Lambda^{-1}\left(\psi^{*} \Lambda \partial_{1} \psi-\Lambda \partial_{1} \psi^{*} \psi\right)
$$

the right-hand side of (9.3) becomes

$$
\frac{1}{\sqrt{g}} e_{1}\left(\sqrt{g} J^{1}\right)=\frac{i}{2 m} q \Lambda^{-1} \partial_{1}\left(\psi^{*} \Lambda \partial_{1} \psi-\Lambda \partial_{1} \psi^{*} \psi\right)
$$

We have here used the relations (5.11). The conservation law becomes then

$$
\partial_{t} \rho=\frac{1}{\sqrt{g}} e_{1}\left(\sqrt{g} J^{1}\right) .
$$

This is the equivalent of (9.3) in the new metric.

It is of interest to compare the structure of the 'space' endowed with the two different metrics we have considered. We saw that the weak completion of the algebra $\mathbb{R}_{q}^{1}$ was in both cases a type- $\mathrm{I}_{\infty}$ factor. The metric can have no effect on this since the set of 'points' is discrete and the induced measures are absolutely continuous one with respect to the other. With the first metric the 'space' is an equally spaced lattice structure within the entire real line. With the second metric one finds a lattice structure with variable spacing within the half-line $(0, \infty)$. In this case it would be natural either to add the origin to obtain a 'space' with boundary or to add the origin and another copy of the 'space' to obtain again the entire real line. In either case the algebra is no longer a factor. It is also to be noticed that the fact we obtained a factor of type $\mathrm{I}_{\infty}$ is due to a choice of representation and not the structure of the algebra. Had we chosen a representation with a continuous spectrum for $\Lambda$ the resulting factor would be of type $\mathrm{II}_{\infty}[23]$.

\section{Acknowledgments}

The authors would like to thank G. Fiore, H. Grosse, L. Vainerman and K. Schmüdgen for enlightening discussions. Two of them $(\mathrm{RH})$ and (JM) would also like to thank the Max-Planck-Institut für Physik in München for financial support.

\section{References}

[1] L.C. Biedenharn, "The quantum group $S U_{q}(2)$ and a $q$-analogue of the boson operators", J. Phys. A: Math. Gen. 22 (1989) L873.

[2] L. Castellani, "Differential Calculus on $I S O_{q}(N)$, Quantum Poincaré algebra and q-Gravity, Commun. Math. Phys. 171 (1995) 383. 
[3] U. Carow-Watamura, M. Schlieker, S. Watamura, W. Weich, "Bicovariant differential calculus on quantum groups $S U_{q}(N)$ and $S O_{q}(N)$ ", Commun. Math. Phys. 142 (1991) 605.

[4] U. Carow-Watamura, M. Schlieker and S. Watamura, " $S O_{q}(N)$ covariant Differential Calculus on Quantum Space and Quantum Deformation of Schrödinger Equation", Z. Phys. C 49 (1991) 439.

[5] A. Connes, "Noncommutative Geometry", Academic Press (1994).

[6] A. Dimakis, J. Madore, "Differential Calculi and Linear Connections", J. Math. Phys. 37 (1996) 4647.

[7] M. Dubois-Violette, R. Kerner, J. Madore, Classical bosons in a noncommutative geometry, Class. Quant. Grav. 6 (1989) 1709.

[8] M. Dubois-Violette, J. Madore, T. Masson, J. Mourad, "Linear Connections on the Quantum Plane", Lett. Math. Phys. 35 (1995) 351.

[9] M. Dubois-Violette, J. Madore, T. Masson, J. Mourad, "On Curvature in Noncommutative Geometry", J. Math. Phys. 37 (1996) 4089.

[10] L.D. Faddeev, N.Y. Reshetikhin and L.A. Takhtajan, "Quantization of Lie Groups and Lie Algebras", Algebra i Analysis, 1 (1989), 178; translation: Leningrad Math. J. 1 (1990), 193.

[11] M. Fichtmueller, A. Lorek, J. Wess, " $q$-deformed Phase Space and its Lattice Structure", Z. Phys. C 71 (1996) 533.

[12] G. Fiore, " $S O_{q}(N)$-isotropic Harmonic Oscillator on the Quantum Euclidean Space $\mathbb{R}_{q}^{N-1}$ ", Proceedings of the Clausthal Symposium on Nonlinear, Dissipative, Irreversible Quantum Systems 1994.

[13] G. Fiore, J. Madore, "Leibniz rules and Reality Conditions", Preprint 98-13, Dip. Matematica e Applicazioni, Università di Napoli, math.QA/9806071.

[14] G. Fiore, J. Madore, "The Geometry of Quantum Euclidean Spaces", LMU Preprint 97/23.

[15] A. Hebecker, S. Schreckenberg, J. Schwenk, W. Weich, J. Wess, "Representations of a q-deformed Heisenberg Algebra", Z. Phys. C 64 (1994) 355.

[16] D. Kasler, J. Madore, T. Masson, "On finite differential calculi", Contemp. Math. 203 (1997) 135.

[17] C. Kittel, "An Introduction to Solid-State Physics", J. Wiley \& Son (1986).

[18] G. Landi, "An Introduction to Noncommutative Spaces and their Geometries", Springer Lecture Notes, Springer-Verlag, (1997).

[19] A. Lorek, A. Ruffing, J. Wess, "A $q$-Deformation of the Harmonic Oscillator", Z. Phys. C 74 (1997) 369.

[20] A.J. Macfarlane, "On $q$-analogues of the quantum harmonic oscillator and the quantum group $S U(2)_{q}$ ", J. Phys. A: Math. Gen. 22 (1989) 4581.

[21] J. Madore, "An Introduction to Noncommutative Differential Geometry and its Physical Applications", Cambridge University Press, (1995).

[22] G.K. Pedersen 1979, " $C^{*}$-algebras and their Automorphism Groups", Academic Press. 
[23] K. Schmüdgen, "Operator representations of a $q$-deformed Heisenberg algebra" Leipzig Preprint 1998, math.QA/9805131.

[24] P. Schupp, P. Watts, B. Zumino, "The 2-Dimensional Quantum Euclidean Algebra", Lett. Math. Phys. 24 (1992) 141.

[25] J. Schwenk, J. Wess, "A q-deformed Quantum Mechanical Toy Model", Phys. Lett. B291 (1992) 273.

[26] J. Schwinger, "Unitary Operator Bases", Proc. Nat. Acad. Sci. 46 (1960) 570.

[27] H.S. Snyder, "Quantized Space-Time”, Phys. Rev. 71 (1947) 38.

[28] J. Wess, B. Zumino, "Covariant differential calculus on the quantum hyperplane", Nucl. Phys. B (Proc. Suppl.) 18 (1990) 302. 